\title{
The influence of topical use of tranexamic acid in reducing blood loss on early operation for thoracolumbar burst fracture: a randomized double-blinded controlled study
}

\author{
Jieliang Shen ${ }^{1} \cdot$ Zhengyang Yang $^{1} \cdot$ Mengyu Fu $^{1} \cdot$ Jie Hao $^{1} \cdot$ Wei Jiang ${ }^{1}$
}

Received: 15 December 2019 / Revised: 2 August 2020 / Accepted: 5 October 2020 / Published online: 24 November 2020

(c) Springer-Verlag GmbH Germany, part of Springer Nature 2020

\begin{abstract}
Purpose To investigate the safety and efficacy of topical use of tranexamic acid (TXA) on early operation for thoracolumbar burst fracture (TBF).

Methods Patients with acute TBF requiring early decompression were prospectively collected. The enrolled patients were randomly assigned to TXA and control group, in which wound surface was soaked with TXA or the same volume of normal saline for $5 \mathrm{~min}$ after wound incision, respectively. The total blood loss (TBL), intraoperative blood loss (IBL), postoperative blood loss (PBL), hemoglobin (HGB) levels on preoperatively (pre-op) and postoperatively, and amount of allogenic blood transfusion were recorded. Furthermore, the general information was also compared between groups.

Results There were 39 and 37 patients enrolled in TXA and control group for final analysis. The demographics data showed no significant difference between groups $(P>0.05)$, but operation time and IBL were significantly decreased in TXA group $(P<0.05)$. Further analysis showed that HGB level was significantly higher in the TXA group at POD1, while the TBL and PBL were significantly less than those in the control group $(P<0.05)$, but similar to HBL $(P>0.05)$. The postoperative ambulation time, removal time of drainage tube, length of hospital stay, and blood transfusion rate were also significantly less in TXA group $(P<0.05)$. At the final follow-up, no neurological deteriorations and no TXA-related complications were observed in both groups.

Conclusion This RCT first demonstrated that topical TXA usage after wound incision could effectively reduce IBL without increasing risk of complications, beneficial to enhanced recovery after early operation for TBF.
\end{abstract}

Keywords Tranexamic acid · Topical use $\cdot$ Blood loss $\cdot$ Early operation · Thoracolumbar burst fracture

\section{Introduction}

Spinal trauma is common in the thoracolumbar (TL) segment due to its location on the junction of spinal biomechanical stress. Major cause for fracture in the TL spine is high-energy trauma, 20 40\% of TL fractures present neurological compression [1]. Thoracolumbar burst fracture

Electronic supplementary material The online version of this article (https://doi.org/10.1007/s00586-020-06626-x) contains supplementary material, which is available to authorized users.

Wei Jiang

390373532@qq.com

1 Department of Orthopedics, The First Affiliated Hospital of Chongqing Medical University, No.1 Youyi Road, Yuzhong District, Chongqing 40042, China
(TBF) is the vertebral segment subjected to axial load on the anterior and middle columns, with posterior wall of the vertebral body protruding into the canal, which may be a cause of neurological injury. There still remains controversial regarding the optimal treatment strategy for TBF. Currently, neurological deficit is deemed as a potential surgical indication to decompress neurologic tissues for recovery [2]. However, the timing of surgical decompression and stabilization is another big controversy that still lacks of strong clinical evidence [3]. It is generally assumed that early surgical intervention may have benefits for reducing the length of stay, intensive care unit stay, ventilator days, morbidity, and hospital expenses within $72 \mathrm{~h}$ of injury [4]. In contrast, other scholars think early surgical intervention may become a "second hit," of which operation for acute spinal fracture is associated with considerably intraoperative bleeding, which not only influences operational manipulation, but 
also adverse to enhanced recovery after surgery (ERAS). Therefore, it seems a safe and efficient treatment strategy to reduce perioperative blood loss when performing an early surgery for TBF.

As a kind of antifibrinolytic agent, tranexamic acid (TXA) has verified its effectiveness in reducing perioperative and traumatic bleeding in various surgeries, of which the efficacy of TXA has also been assessed in spine surgery [5]. A highquality clinical study indicated that TXA could significantly reduce perioperative blood loss in adults undergoing major spinal surgery [6]. However, the concept of major spinal surgery is vague. Furthermore, majority of studies reported the intravenous application of TXA in orthopedic surgery, but systemical TXA usage has potential thrombogenic risk as well as rare but known epileptogenic side effect [7]. Instead, topical use could maintain the local drug concentration by little dosage suggesting well application prospects. There have been many studies demonstrating intravenous or topical administration of TXA could reduce blood loss and allogenic blood transfusions in joint surgery, without a high risk of complications such as deep vein thrombosis (DVT), pulmonary embolism (PE) or wound infection [8, 9]. Recently, some studies have reported the topical usage of TXA in spinal surgery but poorly evaluated, which have relatively small sample sizes as well as some methodological limitations [10]. Therefore, the aim of this prospective randomized double-blinded study was to evaluate the influence of topical TXA usage in reducing blood loss on early operation for TBF with interval time less than $72 \mathrm{~h}$.

\section{Methods}

\section{Patients' data}

Patients with acute TBF and planned to receive operative treatment were prospectively collected at our department from October 2017 to May 2019. Inclusion criteria: (1) age of $18 \sim 50$ years old, no gender preference; (2) bone mineral density detected by dual-energy X-ray absorptiometry $>-1$ $\mathrm{SD}$; (3) identify trauma history, the time of injury $\leq 24 \mathrm{~h}$; (4) single segmental A3 or A4 style fracture located within the TL region (T11 to L2) [11], involved the anterior and middle columns, with retropulsion of posterior bone fragments into the spinal canal as described by Denis [12]; (5) accompanied with neurological deficit, ASIA impairment scale grade $\mathrm{C}$ or D [13]; (6) accompanied with spinal instability (spinal canal encroachment, kyphosis, posterior ligamentous complex injury, et al.), if neurological intact (ASIA grade E). Exclusion criteria: (1) multiple injuries; (2) tumor, infection or osteoporosis induced pathological fracture; (3) complicated with serious underlying diseases, unable to tolerate surgery; (4) history of allergy or hypersensitivity to TXA, current treatment with drugs that interfere with coagulation; (5) coagulation disorders or history of any thromboembolic episode before surgery; (6) anemia before surgery; (7) perioperative cerebrospinal fluid leak. Written informed consent was conventionally signed by all patients at admission.

\section{Trial design}

This is a prospective, randomized, double-blinded clinical trial performed in one hospital. The research approach was approved by the ethics committee of the first affiliated hospital of Chongqing Medical University (2017-100) and registered in chictr.org (ChiCTR-IOR-17013385). The patients were randomized to receive tranexamic acid (TXA group) or saline solution (control group) by a simple, equalprobability randomization scheme. The study had a doubleblinded design with products masked and coded so that the participants were unaware of which product each patient was receiving.

\section{Surgical procedure and interventions}

All the patients enrolled were managed with open decompression, reduction in fracture, and short-segment internal fixation through a standard posterior midline approach. All the operations were performed by one surgeon (H.J) at less than 3 days after initial trauma. In brief, under general anesthesia, patients were positioned pronely and a midline skin incision was made. Subsequently, cut the fascia close to spinous process, strip the sacrospinous muscle along subperiosteum, and expand the muscle laterally to the transverse process. After that, in the TXA group, wound field was soaked with TXA ( $1 \mathrm{~g}$ in $100 \mathrm{ml}$ saline solution) for 5 min, while in the control group, wound field was soaked with the same volume of normal saline. Then, the pedicle screws were implanted into fractured vertebra and the vertebra cephalad and caudad with positions confirmed by $\mathrm{C}$-arm. A unilateral laminectomy was performed to check the protruded bony fragment encroach the canal. Then, place the impactor on the bony fragment and push it down to be decompressed. Set connecting bars; a distraction force was applied using spreader forceps to further restore bony fragment and body height. Ultimately, two ordinary-pressure drainage tubes were placed on both sides of the wound, and a layer-to-layer suture was carried out to close the wound. Patients were instructed to perform isometric contraction and exercise of bilateral lower limb muscles after the operation.

The postoperative drainage $<50 \mathrm{~mL}$ in $24 \mathrm{~h}$ was considered as the standard for the removal of drainage tube, whose removal time was recorded. The postoperative walking ability was assessed by the modified Benzel scale, whose scores ambulation was from I to VII [14]. When the patient 
attained Grade V that represents the ability to walk $7.5 \mathrm{~m}$ with or without assistance, this ambulation time was valid in this study. The ultimate postoperative neurologic status was evaluated at the final follow-up of June 2019.

\section{Outcome evaluation}

The total blood loss (TBL) was estimated according to the formula of Nadler and Gross as follows [15]:(1)preoperative blood volume $(\mathrm{PBV})=\mathrm{k} 1 \times$ height $(\mathrm{m})^{3}+\mathrm{k} 2 \times$ weight $(\mathrm{kg})+\mathrm{k}$ 3 , of which $\mathrm{k} 1=0.3669, \mathrm{k} 2=0.03219, \mathrm{k} 3=0.6041$ for men; $\mathrm{k} 1=0.3561, \mathrm{k} 2=0.03308$, and $\mathrm{k} 3=0.1833$ for women. (2) $\mathrm{TBL}=\mathrm{PBV} \times\left(\mathrm{HCT}_{\text {pre }}-\mathrm{HCT}_{\text {post }}\right) / \mathrm{HCT}_{\text {ave }}$, of which $\mathrm{HCT}_{\text {pre }}$ is the preoperative hematocrit, $\mathrm{HCT}_{\text {post }}$ is the lowest postoperative hematocrit during hospitalization or prior to transfusion, and $\mathrm{HCT}_{\text {ave }}$ is the mean of the $\mathrm{HCT}_{\text {pre }}$ and $\mathrm{HCT}_{\text {post }}$.

Hidden blood loss = TBL-intraoperative blood loss (IBL)postoperative blood loss (PBL), of which IBL was estimated by weighing surgical sponges, measuring blood collected by suction canisters, and subtracting all irrigations fluids added to the surgical field. If the drainage volume was less than $50 \mathrm{~mL} / 24 \mathrm{~h}$, extubation was performed and $\mathrm{PBL}=$ postoperative wound drainage volume $\times$ average of postoperative HCT [16]. The hemoglobin (HGB) was detected preoperatively, and at the first and third day postoperatively (POD1 and POD3). If patient received blood transfusion, the post-transfusion HGB values would not bring into statistical analysis. An allogenic transfusion was given if the HGB level was less than $70 \mathrm{~g} / \mathrm{L}$ or between 70 and $100 \mathrm{~g} / \mathrm{L}$ with anemic symptoms. In addition, the general information including gender, age, body mass index (BMI), duration of operation, ambulation, hospitalization, and neurologic status was also collected.

\section{Statistical analysis}

Statistical analysis was performed by using SPSS software (version 20.0). Quantitative data are presented as median \pm standard deviation. Frequencies and percentages were calculated for qualitative data. Student's t test for continuous data and the $\chi^{2}$ test for categorical data were used for comparisons of variables between the two groups. The level of significance was set at $P<0.05$.

\section{Results}

\section{Baseline characteristics}

Of 124 consecutive patients with TBF, 93 patients were enrolled in this study, and 17 of these patients were subsequently excluded, leaving ultimate 76 patients available for analysis in this study (The detail of CONSORT diagram for this study is found in Fig. 1 and supplementary material). Ultimately, there were 39 patients in the TXA group and 37 patients in the control group for final analysis. The interval time from injury to surgery showed no significant difference between groups, as well as other demographics data (gender, age, BMI, $P>0.05$ ). According to ASIA grades, patients in TXA group presenting C (21), D (13), E (5) were similar with those in control group of which C (19), D (14), E (4), respectively $(P>0.05)$. However, duration of operation and intraoperative blood loss (IBL) were significantly decreased in TXA group $(P<0.05)$ (Table 1$)$.

\section{Blood loss}

Preoperative HGB was similar between the 2 groups $(P>$ $0.05)$, but HGB levels were significantly higher in the TXA group at POD1 $(P<0.05)$, without significant difference at POD3 $(P>0.05)$. Moreover, in the TXA group, the TBL and PBL were significantly less than those in the control group $(P<0.05)$, but there was no significant difference in HBL $(P>0.05)$ (Table 2).

\section{Postoperative condition}

The postoperative ambulation time, removal time of drainage tube, and length of hospital stay were significantly less in the TXA group compared with those in control group ( $P$ $<0.05$ ). One patient receiving TXA required administration of red cell suspension (RCS) postoperatively; in the control group, 5 patients needed postoperative RCS transfusion, of which the blood transfusion rate and volume were significantly lower in the TXA group $(P<0.05)$ (Table 3$)$.

At the final follow-up, no neurologic deteriorations were observed in both groups, who demonstrated an improvement in at least on ASIA grade level from baseline. There were no postoperative complications observed in both groups, such as myocardial infarction, cerebral vascular accident, deep vein thrombosis, pulmonary embolism, wound complications, or hematoma formation within the spinal canal; moreover, none of the TXA-related complications occurred in patients postoperatively, such as seizures, nausea, diarrhea, renal failure.

\section{Discussion}

This is the first double-blinded RCT to evaluate the efficacy and safety of topical TXA application after wound incision for early operation on TBF. The main finding in this study revealed that topical use of TXA had a remarkably decreased volume of TBL, IBL, and PBL, of which the decreased IBL was beneficial for intraoperative manipulation, so as to shorten operation time and promote postoperative recovery. 


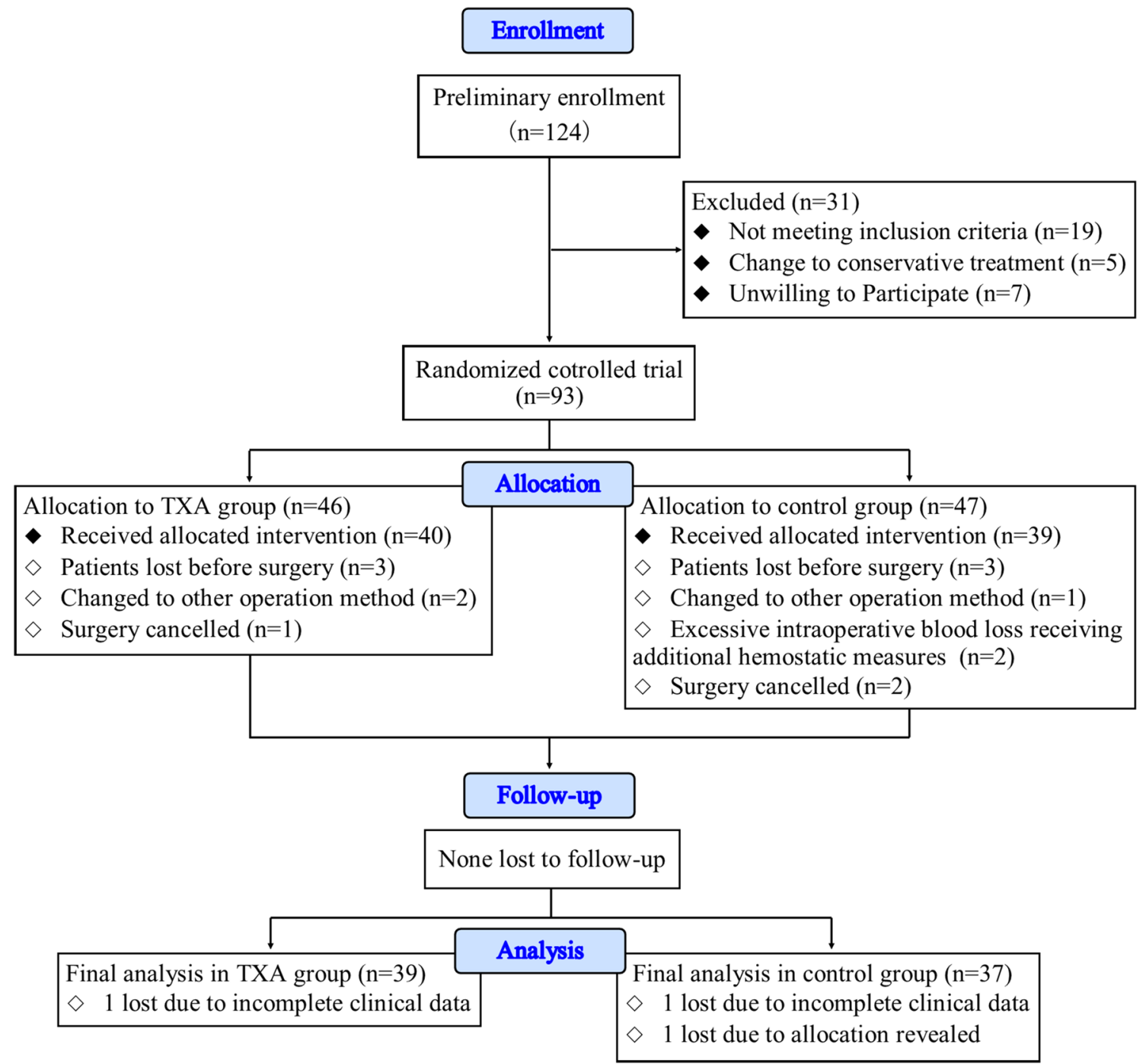

Fig. 1 CONSORT flowchart of patients progress through the study

Table 1 Comparison of baseline characteristics

\begin{tabular}{llll}
\hline Variable & TXA group & Control group & $P$ value \\
\hline $\mathrm{N}$ & 39 & 37 & \\
Interval time (h) & $41.72 \pm 15.83$ & $45.30 \pm 12.86$ & 0.284 \\
Age (years old) & $38.85 \pm 4.17$ & $39.41 \pm 6.51$ & 0.655 \\
Gender (M/F) & $25 / 14$ & $22 / 15$ & 0.814 \\
BMI $\left(\mathrm{kg} / \mathrm{cm}^{2}\right)$ & $26.30 \pm 2.32$ & $25.63 \pm 2.43$ & 0.224 \\
AISA grades & & & \\
$\mathrm{C}$ & 21 & 19 & \\
$\mathrm{D}$ & 13 & 14 & \\
E & 5 & 4 & \\
Intraoperative blood loss & $167.44 \pm 34.01$ & $233.24 \pm 38.81$ & $<0.001$ \\
$\quad(\mathrm{ml})$ & & & \\
Operative duration & $102.18 \pm 11.69$ & $125.49 \pm 11.05$ & $<0.001$ \\
\hline
\end{tabular}

$M$ male; $F$ female
Table 2 Comparison of blood loss

\begin{tabular}{lllr}
\hline Variable & TXA group & Control group & $P$ value \\
\hline HGB level (g/L) & & & \\
Pre-operation & $132.90 \pm 8.41$ & $134.95 \pm 7.00$ & 0.254 \\
POD1 & $115.46 \pm 8.08$ & $106.00 \pm 8.64$ & $<0.001$ \\
POD3 & $122.18 \pm 6.91$ & $120.19 \pm 6.88$ & 0.213 \\
TBL (ml) & $565.38 \pm 32.07$ & $690.54 \pm 52.39$ & $<0.001$ \\
PBL (ml) & $101.28 \pm 14.45$ & $144.05 \pm 30.04$ & $<0.001$ \\
HBL (ml) & $296.67 \pm 27.37$ & $313.24 \pm 50.23$ & 0.082 \\
\hline
\end{tabular}

TXA has been widely applied in clinical practice as a hemostatic agent for bleeding management [17-20]. In a recent high-quality clinical study, TXA has been proved to reduce perioperative blood loss in adults undergoing major spinal surgery [6]. However, multiple factors contribute to 
Table 3 Comparison of postoperative condition

\begin{tabular}{lllc}
\hline Variable & TXA group & Control group & $P$ value \\
\hline Blood transfusion & & & \\
Transfusion rate (\%) & $2.56(1 / 39)$ & $13.51(5 / 37)$ & $<0.001$ \\
Transfusion volume (ml) & 200 & 1000 & - \\
Ambulation time (day) & $3.38 \pm 0.82$ & $7.00 \pm 2.91$ & $<0.001$ \\
Removal time of drainage tube (day) & $2.26 \pm 0.79$ & $2.97 \pm 0.73$ & $<0.001$ \\
Length of hospital stay (day) & $6.08 \pm 0.77$ & $9.78 \pm 2.03$ & $<0.001$ \\
\hline
\end{tabular}

the complex causes of bleeding in spinal surgery, of which disease category and surgical procedure play crucial roles. Take decompression surgery, for example; the amount of bleeding for resection of a spinal metastatic tumor or a disc varies greatly; similarly, for a lumbar disc herniation, the influence of percutaneous endoscopic discectomy or open discectomy on blood loss must be an enormous difference. Therefore, the enrolled disease category (one-level vertebral fracture for early operation) and surgical procedure (posterior short-segment fixation and unilateral laminectomy for direct decompression) were strictly limited in this study, to reduce analytical bias for the influence of topical use of TXA in reducing blood loss.

TXA has been typically administered intravenously throughout the surgery. A latest meta-analysis suggests that only high-dose intravenous TXA can significantly reduce intraoperative-perioperative allogenic transfusion rates and operative time, whereas low dose cannot [10]. However, systemic administration of TXA can have potential adverse effects on patients with clotting disorders or renal impairment. Moreover, the fact that excessive fibrinolysis as a result of acute consumptive coagulopathy from surgical trauma is most severe in the surgical wound itself and often persists throughout the postoperative period has pushed topical application of TXA to improve the current standard of care for hemostasis in surgery [21]. Compared with intravenous TXA application, the topical TXA application has the advantages of the maximum concentration at the bleeding field and avoiding systemic exposure of TXA. A favorable clinical safety has been proved in this study; no TXA-associated complications were recorded. Wang et al. suggested that intraoperative topical application of TXA before wound closure is not recommended for TL fracture [22]. Further analysis would indicate that they treated TL fracture through percutaneous pedicle screw fixation whose small percutaneous fixation wound led to little bleeding. It is unable to fully reflect the hemostatic effect of TXA in their minimally invasive surgery. In this study, the open short-segment decompression and fixation were underwent for TBF, whose mean blood loss volume in previous studies was roughly from $200 \sim 300 \mathrm{ml}[23,24]$. This reported intraoperative blood loss was similar as our result in control group, which demonstrated that the significant reduction in blood loss for the TXA treatment was not because the blood loss in the control group was artificially higher than the loss seen in regular practice, but due to better hemostatic effect of topical TXA usage.

TBF is usually caused by high-energy damage, and the coagulopathy of trauma is a complex pathophysiologic state that results in diffuse, microvascular bleeding [25]. In consequence, the bleeding risks of early operation of open decompression for TBF are plasminogen system activation, vertebralis injury, and cancellous bleeding. TXA is an antifibrinolytic lysine analogue that competitively inhibits plasminogen, plasmin, and tissue plasminogen activator at lysine binding sites [26]. In previous studies, the applied occasion of TXA was usually before wound closure; however, in this way it made no contribution to reducing bleeding during operative procedure that would not obviously affect IBL. Therefore, we moved the applied occasion forward to after opening the wound. In this way, topical use of TXA not only significantly reduced bleeding from surrounding soft tissue and venae vertebralis to make a dry operative field for visibility, but also was effective to decrease blood loss from cancellous bone especially during laminectomy. The bleeding from exposed bony surfaces is not amenable to standard hemostatic maneuvers that can continue even after the wound is closed [27]. But TXA can decrease this type of blood loss by attenuating the enhanced fibrinolytic activity that has been shown to occur in spine surgery. Hence, accompanied with decreased IBL, the TXA group showed significantly less PBL, removal time of drainage tube, and length of hospital stay, indicating faster recovery after surgery [28].

Further analysis showed that HGB level was significantly higher in the TXA group on POD1. In spite of no significant difference being found on POD3, the postoperative blood transfusion significantly reduced in TXA group, which decreased the risk of transfusion-related complications. Although Colomina et al. reported that TXA did not significantly reduce transfusion requirements in major spinal surgery, their homogeneity of surgical approach and techniques were poor [6]. Interestingly, the TBL, IBL, and PBL in TXA group were significantly less than those in control group, but the HBL showed no difference between groups in this study. The potential reason may be HBL was calculated by 
the formula without direct measurement, and postoperative adequate drainage rendered the volume of HBL decreased significantly in both groups to largely close the gap.

\section{Conclusion}

Previous researches have substantial differences in disease categories, surgical procedures, application forms, and dose to explore the safety and efficacy of TXA on spinal surgery, which lead to high heterogeneity of research results. The strength of our study is the homogeneity of enrolled patients receiving the same operation by the same surgeon at the same hospital. The result of this study provides a safe and efficient treatment strategy for early operation on TBF that topical use of TXA after wound incision can effectively reduce IBL without increased risk of complications, beneficial to enhanced postoperative recovery.

\section{Compliance with ethical standards}

Conflict of interest The authors declare that they have no confict of interest.

\section{References}

1. AbdelFatah MA (2017) The walking recovery one year after surgical management of thoracolumbar burst fracture in paraplegic patients. Neurol Med Chir 57:467-471. https://doi.org/10.2176/ nmc.oa.2017-0017

2. Abeysiri S, Chau M, Highton D, Richards T (2019) Management of the patient presenting with anaemia in the preoperative setting. Transfus Apher Sci 58:392-396. https://doi.org/10.1016/j.trans ci.2019.06.014

3. Ali ZS, Ma TS, Ozturk AK, Malhotra NR, Schuster JM, Marcotte PJ, Grady MS, Welch WC (2018) Pre-optimization of spinal surgery patients: development of a neurosurgical enhanced recovery after surgery (ERAS) protocol. Clin Neurol Neurosurg 164:142-153. https://doi.org/10.1016/j.clineuro.2017.12.003

4. Beyda R, Johari D (2019) Tranexamic acid for upper gastrointestinal bleeding. Acad Emerg Med 26:1181-1182. https://doi. org/10.1111/acem.13835

5. Brohi K, Cohen MJ, Davenport RA (2007) Acute coagulopathy of trauma: mechanism, identification and effect. Curr Opin Crit Care 13:680-685. https://doi.org/10.1097/MCC.0b013e3282f1e78f

6. Cahueque M, Cobar A, Zuniga C, Caldera G (2016) Management of burst fractures in the thoracolumbar spine. J Orthop 13:278281. https://doi.org/10.1016/j.jor.2016.06.007

7. Colomina MJ, Koo M, Basora M, Pizones J, Mora L, Bago J (2017) Intraoperative tranexamic acid use in major spine surgery in adults: a multicentre, randomized, placebo-controlled trialdagger. Br J Anaesth 118:380-390. https://doi.org/10.1093/bja/aew43 4

8. Denis F (1983) The three column spine and its significance in the classification of acute thoracolumbar spinal injuries. Spine 8(8):817-831. https://doi.org/10.1097/00007632-19831 $1000-00003$
9. Egea-Guerrero JJ, Ballesteros MA, Quintana-Diaz M (2019) Tranexamic acid in patients with multiple injuries: good, elegant, and cheap? Emergencias 31:281-282

10. Fahrenholtz CG, Bonanno LS, Martin JB (2019) Tranexamic acid as adjuvant treatment for postpartum hemorrhage: a systematic review protocol. JBI Database Syst Rev Implement Rep 17:15651572. https://doi.org/10.11124/JBISRIR-2017-003978

11. George J, Eachempati KK, Subramanyam KN, Gurava Reddy AV (2018) The comparative efficacy and safety of topical and intravenous tranexamic acid for reducing perioperative blood loss in total knee arthroplasty - a randomized controlled non-inferiority trial. Knee 25:185-191. https://doi.org/10.1016/j.knee.2017.11.006

12. Ipema HJ, Tanzi MG (2012) Use of topical tranexamic acid or aminocaproic acid to prevent bleeding after major surgical procedures. Ann Pharmacother 46:97-107. https://doi.org/10.1345/ aph.1Q383

13. Lin CL, Chou PH, Fang JJ, Huang KY, Lin RM (2018) Short-segment decompression and fixation for thoracolumbar osteoporotic fractures with neurological deficits. J Int Med Res 46:3104-3113. https://doi.org/10.1177/0300060518772422

14. Oner C, Rajasekaran S, Chapman JR, Fehlings MG, Vaccaro AR, Schroeder GD, Sadiqi S, Harrop J (2017) Spine trauma-what are the current controversies? J Orthop Trauma 31(Suppl 4):S1-S6. https://doi.org/10.1097/BOT.0000000000000950

15. Raman T, Varlotta C, Vasquez-Montes D, Buckland AJ, Errico TJ (2019) The use of tranexamic acid in adult spinal deformity: is there an optimal dosing strategy? Spine J 19:1690-1697. https:// doi.org/10.1016/j.spinee.2019.06.012

16. Roberts TT, Leonard GR, Cepela DJ (2017) Classifications in brief: American spinal injury association (Asia) impairment scale. Clin Orthop Relat Res 475:1499-1504. https://doi.org/10.1007/ s11999-016-5133-4

17. Rosenthal BD, Boody BS, Jenkins TJ, Hsu WK, Patel AA, Savage JW (2018) Thoracolumbar burst fractures. Clin Spine Surg 31:143-151. https://doi.org/10.1097/BSD.0000000000000634

18. Samson AL, Alwis I, Maclean JAA, Priyananda P, Hawkett B, Schoenwaelder SM, Jackson SP (2017) Endogenous fibrinolysis facilitates clot retraction in vivo. Blood 130:2453-2462. https:// doi.org/10.1182/blood-2017-06-789032

19. Schnake KJ, Schroeder GD, Vaccaro AR, Oner C (2017) AOSpine classification systems (subaxial, thoracolumbar). J Orthop Trauma 31(Suppl 4):S14-S23. https://doi.org/10.1097/BOT.0000000000 000947

20. Shin SR, Lee SS, Kim JH, Jung JH, Lee SK, Lee GJ, Ju Moon B, Lee JK (2020) Thoracolumbar burst fractures in patients with neurological deficit: anterior approach versus posterior percutaneous fixation with laminotomy. J Clin Neurosci 75:11-18. https:// doi.org/10.1016/j.jocn.2020.03.046

21. Theusinger OM, Spahn DR (2016) Perioperative blood conservation strategies for major spine surgery. Best Pract Res Clin Anaesthesiol 30:41-52. https://doi.org/10.1016/j.bpa.2015.11.007

22. Wanderman N, Robinson W, Carlson B, Bydon M, Currier B, Huddleston P, Nassr A, Freedman B (2019) Tranexamic acid in thoracic and lumbar fusions and perioperative blood loss. Clin Spine Surg 32:E462-E468. https://doi.org/10.1097/BSD.00000 00000000851

23. Wang X, Yang R, Sun H, Zhang Y (2019) Different effects of intravenous, topical, and combined application of tranexamic acid on patients with thoracolumbar fracture. World Neurosurg 127:e1185-e1189. https://doi.org/10.1016/j.wneu.2019.04.095

24. Xie J, Hu Q, Huang Q, Ma J, Lei Y, Pei F (2017) Comparison of intravenous versus topical tranexamic acid in primary total hip and knee arthroplasty: an updated meta-analysis. Thromb Res 153:28-36. https://doi.org/10.1016/j.thromres.2017.03.009

25. Xing D, Chen Y, Ma JX, Song DH, Wang J, Yang Y, Feng R, Lu J, Ma XL (2013) A methodological systematic review of early versus 
late stabilization of thoracolumbar spine fractures. Eur Spine J 22:2157-2166. https://doi.org/10.1007/s00586-012-2624-1

26. Xu D, Zhuang Q, Li Z, Ren Z, Chen X, Li S (2017) A randomized controlled trial on the effects of collagen sponge and topical tranexamic acid in posterior spinal fusion surgeries. J Orthop Surg Res 12:166. https://doi.org/10.1186/s13018-017-0672-2

27. Yerneni K, Burke JF, Tuchman A, Li XJ, Metz LN, Lehman RA Jr, Lenke LG, Tan LA (2019) Topical tranexamic acid in spinal surgery: a systematic review and meta-analysis. J Clin Neurosci 61:114-119. https://doi.org/10.1016/j.jocn.2018.10.121

28. Yin M, Chen G, Yang J, Tong Z, Xu J, Huang Q, Ma J, Mo W (2019) Hidden blood loss during perioperative period and the influential factors after surgery of thoracolumbar burst fracture: a retrospective case series. Medicine 98:e14983. https://doi. org/10.1097/MD.0000000000014983

Publisher's Note Springer Nature remains neutral with regard to jurisdictional claims in published maps and institutional affiliations. 